\title{
Powerful femtosecond singular pulses in the Kerr media with dispersion
}

\author{
${ }^{1}$ Khasanov O., ${ }^{2}$ Smirnova T., ${ }^{1}$ Fedotova O., ${ }^{1}$ Rusetsky G. and ${ }^{3,4}$ Volyar A. \\ ${ }^{1}$ Scientific-Practical Material Research Centre of the Belarus National Academy of \\ Sciences, 19 P. Brovki St., 220072 Minsk, Belarus, khasanov@ifttp.bas-net.by \\ ${ }^{2}$ International Sakharov Environmental University, 23 Dolgobrodskaya St., 220009 \\ Minsk, Belarus \\ ${ }^{3}$ Physical Department of National Taurida V. Vernadsky University, 4 Yaltinskaya \\ St., Simferopol, 95007 Crimea, Ukraine \\ ${ }^{4}$ Institute of Physical Optics, 23 Dragomanov St., 79005 Lviv, Ukraine
}

Received: 19.12 .2012

\begin{abstract}
Nonlinear dynamics of high-intensity femtosecond singular pulses in a Kerr medium with group velocity dispersion (GVD) is analysed using variational and numerical approaches. We study underlying physical processes governing propagation of vortices: diffraction, Kerr self-focusing, defocusing due to photoinduced plasma, and normal/anomalous GVD. Multifoci behaviour of vortices appearing in the medium are revealed. It is shown that the refocusing period depends on both the magnitude and sign of the GVD. The early stages of vortex beam filamentation are described.
\end{abstract}

Keywords: femtosecond pulsed beam, optical vortex, topological charge, group velocity dispersion, Kerr nonlinearity, multiphoton ionisation, free-electron plasma.

PACS: $42.65 .-\mathrm{k}$

UDC: 535.18

\section{Introduction}

After introduction by Nye and Berry in 1974 [1], optical vortices have become interesting subjects of research [2] and possible applications. Optical vortices have large potentials for applications in such fields as, e.g., information processing [3], optical trapping of particles and transfer of orbital angular momentum [4], and soliton algebra [5]. Beyond doubt, stability of vortices represents an important issue for both linear [6] and nonlinear [7] propagation regimes. This problem has drawn much attention of researchers because sometimes only specific design of samples or distinctive conditions can provide stability of propagating vortices [6].

In our previous work [8] we have investigated the propagation of highly intense femtosecond singular pulses in a Kerr medium, depending on the topological charge and the ratio of initial and critical pulse powers. We have found that the underlying physical mechanism for stable vortex propagation is dynamic competition between a Kerr self-focusing and a defocusing caused by plasma due to multi-photon ionisation processes. For the first time the role of inertial origin of plasma formation has been analysed, which provides stable propagation of the femtosecond vortices. The stability of the vortex beam against azimuthal perturbations has been elucidated, too. The main results obtained in the study [8] may be formulated as follows. It is the competition of the focusing and defocusing factors at weak dissipation and especially the inertial character of plasma that can provide a quasi-soliton regime of propagation for the pulsed femtosecond vortex beams. Moreover, the singly charged vortex is stable at the scales exceeding three diffraction 
lengths or even more, which are much longer than those for the hypothetic case of instantaneous character of the plasma. The similar scenarios take place for the vortices with larger topological charges, though the distances of vortex stability become essentially shorter.

Below we will dwell on the main peculiarities of nonlinear dynamics of high-intensity femtosecond singular pulses in Kerr dielectrics, taking into account the effect of group velocity dispersion (GVD). The impact of the GVD on the vortex-beam stability becomes very important in optical fibres and photonic crystals, as well as for ultra-short singular pulses. Our analysis will include variational and numerical approaches.

\section{Basic equations}

Let us consider the behaviour of powerful femtosecond vortex pulse with the topological charge $m$ in a fused silica. The underlying process providing a possible stable propagation of the vortex in the medium is the competition of cubic focusing nonlinearity and defocusing caused by plasma generation. The pulse propagation along some $z$ axis is described by a set of equations, which consists of a generalised nonlinear Schrödinger equation for the complex envelope $E=E(r, t, z)$ of the electric field and a kinetic equation for the free-electron density $\rho$ :

$$
\begin{gathered}
\frac{\partial E}{\partial z}=\frac{i}{2 k} \nabla_{\perp}^{2} E-i \frac{\beta_{2}}{2} \frac{\partial^{2} E}{\partial t^{2}}+i k_{0} n_{2}|E|^{2} E-\frac{\sigma}{2}\left(1+i \omega_{0} \tau_{c}\right) \rho E-\frac{\beta^{(K)}}{2}|E|^{2 K-2} E \\
\frac{\partial \rho}{\partial t}=\frac{\beta^{(K)}}{K \hbar \omega}|E|^{2 K}-\frac{\rho}{\tau_{r}} .
\end{gathered}
$$

Regarding the notations, $\beta_{2}$ is the GVD coefficient, $n_{0}$ and $n_{2}$ respectively the linear and nonlinear refractive indices for the fused silica, $k_{0}=\omega / c, n_{0}=1.45, n_{2}=3 \times 10^{-16} \mathrm{~cm}^{2} / \mathrm{W}$, $\sigma=1.55 \times 10^{-18} \mathrm{~cm}^{2}$ [9] denotes the inverse bremsstrahlung cross section, $\tau_{c}=2.33 \times 10^{-14} \mathrm{~s}$ the electron collision time, $\tau_{r}=300 \times 10^{-15} \mathrm{~s}$ the electron recombination time resulting from electronphonon interaction, and $\beta^{(K)}$ the Keldysh parameter for the K-photon absorption defined as [9]

$$
\beta^{(K)}=\frac{2}{9 \pi} \omega\left(\frac{m_{e} \omega}{\hbar}\right)^{3 / 2}\left(\frac{q_{e}^{2}}{16 m_{e} E_{g} \omega^{2}}\right)^{K} e^{2 K} \Phi(s), \Phi(s)=e^{-s^{2}} \int_{0}^{s} e^{-x^{2}} d x, s=\sqrt{2 K-\frac{2 E_{g}}{\hbar \omega}} .
$$

Here $m_{e}$ and $q_{e}$ are respectively the electron mass and the elementary charge, $E_{g}=9.1 \mathrm{eV}$ means the band gap, and $K=\left[\frac{E_{g}}{\hbar \omega}\right]+1$ the number of photons in the multiphoton absorption process.

For the fused silica at the wavelength $\lambda=800 \mathrm{~nm}$ we have $K=6, \beta_{2}=361 \mathrm{fs}^{2} / \mathrm{cm}$, and $\beta^{(K)}=6.49 \times 10^{-104} \mathrm{~m}^{9} /\left(\mathrm{s} \mathrm{W}^{6}\right)$. In other words, we assume that the plasma is formed due to multiphoton ionisation mechanism, as the latter is indeed the most essential under the conditions specified above. Different terms appearing in the r. h. s. of Eq. (1) describe the diffraction, the GVD, the cubic nonlinearity, the plasma defocusing, and the multiphoton absorption. The first term in the r. h. s. of Eq. (2) is responsible for the multiphoton ionisation and the second one accounts for the electron recombination. Provided that the propagating pulse duration is far shorter than the electron-phonon relaxation time, the effect of recombination can be neglected in the subsequent discussion.

Ukr. J. Phys. Opt. 2013, Volume 14, Issue 2 
Let the light pulse with the wavelengths $\lambda=800 \mathrm{~nm}$ or $1500 \mathrm{~nm}$ and the duration $\tau_{p}=10$ $120 \mathrm{fs}$ pass through the bulk fused silica. This pulse should be susceptible to influence of the GVD. We assume that the input pulse intensity $I_{0}$ varies in the range of $1-70 \mathrm{TW} / \mathrm{cm}^{2}$ and the beam waist $w_{0}$ can acquire the values between $12 \mu \mathrm{m}$ and $50 \mu \mathrm{m}$. We consider the propagation of a doughnut-shaped pulsed beam carrying the vortex with the topological charge $m$.

We choose the boundary conditions for the field amplitude as follows:

$$
\begin{gathered}
E(z=0)=\sqrt{\frac{2^{m}}{m ! \pi a_{0}^{2} T_{0}}} E_{0} \frac{r^{|m|}}{a_{0}^{m}} \exp \left(\frac{-r^{2}}{a_{0}^{2}}-\frac{t^{2}}{T_{0}^{2}}\right) \exp (\operatorname{im} \varphi), \\
\frac{\partial E(r=0)}{\partial r}=E(r=\rightarrow \infty)=0
\end{gathered}
$$

Let us introduce the dimensionless variables $\bar{z}=z / L_{d f}, \bar{r}=r / w_{0}$ and $\bar{t}=(t-z / v) / \tau_{p}$, where $\bar{z}$ and $\bar{r}$ are the dimensionless longitudinal and lateral variables, $\bar{t}$ implies the dimensionless time in the frame of reference moving with the group velocity $v, L_{d f}=k w_{0}^{2} / 2$ the diffraction length, and $k=\omega n_{0} / c$. The dimensionless field amplitude is $e=E / E_{0}$, with $E_{0}$ being the input peak field amplitude. Then Eq. (1) in the dimensionless form reads as

$$
, \frac{\partial e}{\partial z}=i \Delta e-i \delta \frac{\partial^{2} e}{\partial \bar{t}^{2}}+8 i \alpha|e|^{2} e-\frac{\sigma}{2}\left(1+i \omega \tau_{c}\right) e-\mu_{K}|e|^{2 K-2} e-i \frac{m^{2}}{\bar{r}^{2}} e,
$$

with $\alpha=\frac{P_{i n}}{P_{c r}}, P_{i n}=\frac{w_{0}^{2} n_{0} c\left|E_{0}\right|^{2}}{2}, P_{c r}=\frac{\lambda^{2}}{2 \pi n_{0} n_{2}}, \delta=\frac{k w_{0}^{2}}{4} \frac{\beta_{2}}{\tau_{p}^{2}}$, and $\mu_{K}=\frac{k w_{0}^{2}}{4} \beta^{(K)}\left|\frac{2^{m+1} P_{i n}}{\pi m ! w_{0}^{2}}\right|^{K-1}$. Eq. (2) can be reduced to its dimensionless form in an analogous manner.

\section{Two-scale variational approach}

In this section we present the results of variational analysis of the set of Eqs. (1) and (2). The variational approach represents only an approximation, being good enough as an initial trial solution. However, its advantage is that it provides a semi-analytical result, which can easily be analysed, and often gives a simple physical insight to the origin of processes [10-12]. Let us use the ansatz

$$
E(r, z, t)=A(r, z, t) \exp \{i \varphi(r, z, t)\},
$$

where $A(r, z, t)$ is the amplitude of the pulse and its phase $\varphi(r, z, t)$ is given by

$$
\varphi(r, z, t)=\psi(r, z, t)+m \theta \text {. }
$$

Substituting Eqs. (5) and (6) into Eq. (1), we arrive at the set of equations for the wave amplitude and phase:

$$
\begin{aligned}
& \frac{\partial A}{\partial z}+\frac{1}{k} \frac{\partial A}{\partial r} \frac{\partial \psi}{\partial r}+\frac{1}{2 k} A \frac{\partial^{2} \psi}{\partial r^{2}}+\frac{1}{2 k} \frac{A}{r} \frac{\partial \psi}{\partial r}-\beta_{2} \frac{\partial A}{\partial t} \frac{\partial \psi}{\partial t}-\frac{\beta_{2}}{2} \frac{\partial^{2} \psi}{\partial t^{2}}+\frac{\sigma}{2} \rho A+\frac{\beta^{(K)}}{2} A^{2 K-1}=0 \\
& \frac{\partial \psi}{\partial z}+\frac{1}{2 k}\left(\frac{\partial \psi}{\partial r}\right)^{2}-\frac{\beta_{2}}{2}\left(\frac{\partial \psi}{\partial t}\right)^{2}-\frac{1}{2 k} \frac{1}{A}\left(\frac{\partial^{2} A}{\partial r^{2}}+\frac{1}{r} \frac{\partial A}{\partial r}\right)+\frac{\beta_{2}}{2} \frac{1}{A} \frac{\partial^{2} A}{\partial t^{2}}-k_{0} n_{2} A^{2} \\
&+\frac{1}{2} \sigma \omega_{0} \tau_{c} \rho+\frac{1}{2 k} \frac{m^{2}}{r^{2}}=0
\end{aligned}
$$


Using a classical-mechanics analogy of a particle moving in two-dimensional potential well with regard to Eq. (8), one obtains the Hamilton function

$$
H=\frac{1}{2 k}\left(\frac{\partial \psi}{\partial r}\right)^{2}-\frac{\beta_{2}}{2}\left(\frac{\partial \psi}{\partial t}\right)^{2}-\frac{1}{2 k}\left(\frac{1}{A} \frac{\partial^{2} A}{\partial r^{2}}+\frac{1}{A r} \frac{\partial A}{\partial r}-\frac{\beta_{2} k}{2} \frac{\partial^{2} A}{\partial t^{2}}\right)+2 k k_{0} n_{2} A^{2}-k \sigma \omega_{0} \tau \rho \rho-\frac{m^{2}}{r^{2}} .
$$

Following the analogy suggested in the work [13] for the Gaussian beams, we have developed the Hamiltonian approach to the pulsed vortex beam in order to get equations for its radius and duration. Indeed, having known the potential function $V(r, t)$ depending on the two variables $r$ and $t$,

$$
V(r, t)=-\frac{1}{2 k}\left(\frac{1}{A} \frac{\partial^{2} A}{\partial r^{2}}+\frac{1}{A r} \frac{\partial A}{\partial r}-\frac{\beta_{2} k}{2} \frac{\partial^{2} A}{\partial t^{2}}\right)+2 k k_{0} n_{2} A^{2}-k \sigma \omega_{0} \tau_{c} \rho-\frac{m^{2}}{r^{2}}
$$

it is easy to get an equation of motion for them:

$$
\frac{d^{2} r_{i}}{d z^{2}}=-\frac{1}{k} \frac{\partial V}{\partial r_{i}}
$$

Here we take $z, r, t$ and $k$ in Eq. (8) as $t, q_{1}, q_{2}$ and $m$ in the Hamilton-Jacobi equation, which describes particle's movement in a two-dimensional potential well in the classical mechanics. Note that $r$ and $t$ are the radial and temporal distances of some arbitrary light ray in the pulsed beam from the centre of the latter, which is specified by the conditions $r=0$ and $t=0$. The problem under consideration reduces to solution of equations for $r$ and $t$ with Eq. (7), though the solution can be notably simplified in the frame of aberrationless approximation. We assume surely enough that the pulsed vortex beam retains a doughnut form:

$$
A(r, z, t)=\sqrt{\frac{2^{m}}{m !} \frac{P_{i n}}{\pi a^{2}(z) T(z)}} \frac{r^{m}}{a^{m}(z)} \exp \left(-\frac{r^{2}}{a^{2}(z)}-\frac{t^{2}}{T^{2}(z)}\right),
$$

where $a(z)$ and $T(z)$ are the beam radius and its duration, respectively. Being interested in the behaviour of the waist and the temporal width of the singular pulse, we can arrive at the following system of equations for $a(z)$ and $T(z)$ in the aberrationless approximation:

$$
\begin{aligned}
& \frac{\mathrm{d}^{2} a}{\mathrm{~d} z^{2}}=\frac{1}{k^{2}}\left[\frac{4}{a^{3}}+2 k k_{0} n_{2} A^{2}(a, T) \frac{(m-2)}{a}-k \sigma \omega_{0} \tau_{c} K \rho(a, T) \frac{(m-2)}{a}\right], \\
& \frac{\mathrm{d}^{2} T}{\mathrm{~d} z^{2}}=2 \beta_{2}\left(\frac{2 \beta_{2}}{T^{3}}+2 k_{0} n_{2} \frac{A^{2}(a, T)}{T}-\frac{\sigma \omega_{0} \tau_{c}}{4} \frac{\partial \rho(a, T)}{\partial T}\right),
\end{aligned}
$$

The system of Eqs. (13) and (14) is integro-differential, since the plasma density represents an integral value. For the topological charge $m=0$, these equations are similar to those for the transverse and temporal mean-square widths of the Gaussian beam centred at the origin $t=0$ and $r=0$, which has been obtained in Ref. [11] in a different way. In the absence of plasma contribution our equations reduce to those obtained for the pulsed beam's width and duration in the work [10], the authors of which have considered spatiotemporal effects at the vortex-free pulse propagation in the dispersive medium with the Kerr nonlinearity. Using a simple integration rule, the electron density can be approximated as $\rho(t)=\frac{g(t) \beta^{(K)}}{K \hbar \omega}|A|^{2 K}$ while integrating up to the pulse peak, with $g(t)=0.5\left(t_{\min }+t\right)$ and $t_{\text {min }}$ denoting the cutoff time determined by the parameters of the 
initial pulse [12]. It is important that the electron density has to be evaluated for each propagation distance, which does not pertain in this case. However, most of the important physics is still retained in this approximation. Moreover, the approximation not only simplifies the calculations but also makes the interpretation more transparent.

The analysis of Eq. (13) with regard to dependences on the input parameters of the beam reveals periodic behaviour of both the waist and the intensity of the pulse propagating as far as several diffraction lengths, which is due to the competition of the focusing and defocusing effects. The refocusing period (RP) is determined by the pulse parameters. These are the intensity and the radius of the pulse, the ratio of the input pulse power to the critical (threshold) self-focusing power, and also the input pulsed-beam collimating parameter $(\mathrm{d} a / \mathrm{d} z)_{0}=(\mathrm{d} a / \mathrm{d} z)$ for $z=0$. According to our variational analysis, the less radius and the higher intensity of the input pulse the less the RP becomes. Moreover, this period depends on the pulse duration: the less duration, the less RP is. This originates from higher peak intensities of shorter pulses. Fig. 1 demonstrates behaviour of the pulse radius and duration when the GVD is negligible. In this case the radius oscillates with a constant period and every time after refocusing stage it acquires its original value under propagation free of dissipation. As seen from Fig. 1, the pulse duration is constant under these conditions and does not change along the propagation axis.

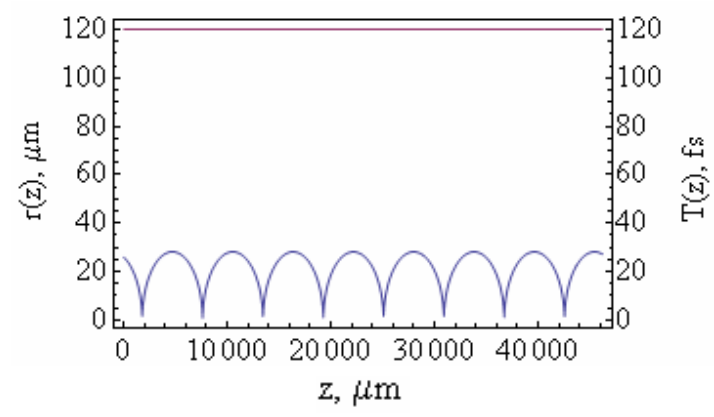

Fig. 1. Spatial behaviours of transverse radius $r(z)$ (lower curve) and duration $T(z)$ (upper curve) of doughnut pulsed beam in the fused silica along the propagation axis $z$ for $m=1$ and the initial parameters $(\mathrm{d} a / \mathrm{d} z)_{0}=-0.0041, a_{0}=26 \mu \mathrm{m}, T_{0}=120 \mathrm{fs}$, $I_{0}=6.19 \mathrm{TW} / \mathrm{cm}^{2}$, and $\alpha=31.13$.
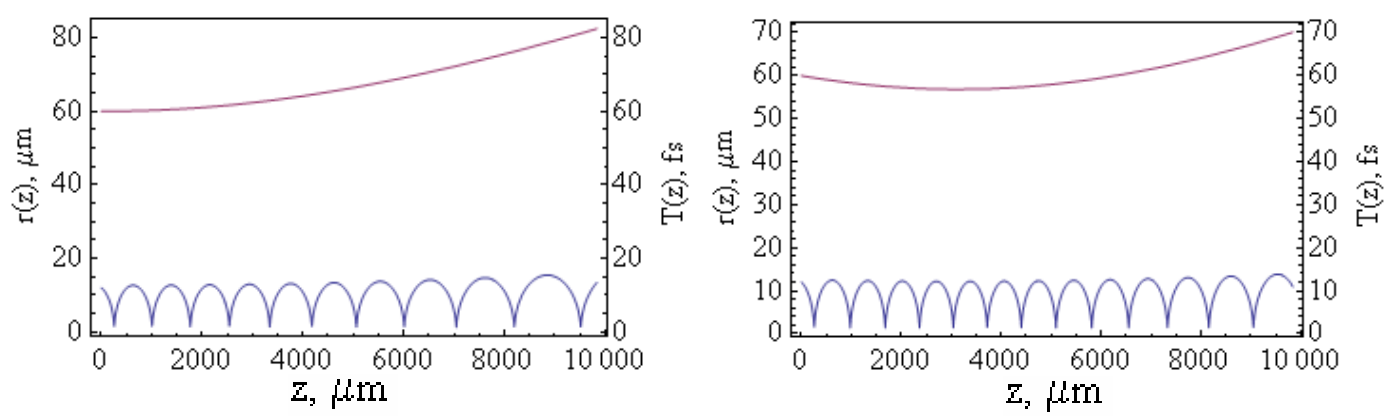

Fig. 2. Impact of normal GVD on pulse duration $T(z)$ (upper curves) and radius $r(z)$ (lower curves) under propagation in the fused silica for $m=1, \alpha=62.265$ and the input parameters $a_{0}=12 \mu \mathrm{m}, T_{0}=60 \mathrm{fs}$, $\lambda=800 \mathrm{~nm}, \mathrm{da} / \mathrm{d} z(z=0)=-0.011, I_{0}=58.2 \mathrm{TW} / \mathrm{cm}^{2}$, and $\beta_{2}=361 \mathrm{fs}^{2} / \mathrm{cm}:(a) \mathrm{d} T / \mathrm{d} z(z=0)=-0.0001$ and (b) $\mathrm{d} T / \mathrm{d} z(z=0)=-0.02$.

Different scenarios of nonlinear dynamics of the pulse are realised with increasing GVD. Our simulations demonstrate a strong dependence of behaviour of the singly charged vortex on both the magnitude and sign of the GVD. In particular, Fig. 2a elucidates dependences of the temporal and spatial widths of the pulse upon the distance, which are characterised by beam radius oscillations and by steady trend of increasing the temporal width under complete passage of the pulse. 
Stretching of the pulse along the propagation axis is accompanied by increasing its spatial width and the RP. It should be noticed that this result contradicts the data of the work [14].

As shown in [14], the femtosecond vortex-free pulse shortens significantly along the propagation axis under the combined influence of diffraction, Kerr nonlinearity, normal GVD and photoinduction of plasma. Then the compression factor can reach the value of 5.5. According to our data, the influence of plasma nonlinearity is quite weak and it cannot compensate for the normal GVD effect and make the vortex pulse shorten. Higher plasma density and the input pulse chirp $\mathrm{d} T / \mathrm{d} z(z=0)$ can reverse the pulse elongation process. One can see from Fig. $2 \mathrm{~b}$ that the vortex beam with $\mathrm{d} T / \mathrm{d} z(\mathrm{z}=0)=-0.02$ in the fused silica self-focuses and shortens up to $z=4 \mathrm{~mm}$ and then its RP and temporal width increase.

Dynamics of the vortex pulse depends not only on the GVD value but on its sign. The pulse shortens along the propagation axis in the media with anomalous dispersion. This is accompanied by periodic self-focusing and defocusing of the pulse. The mechanism underlying this stretching process may be explained in terms of competition of the anomalous GVD and the Kerr focusing. The smaller the temporal width of the pulse, the smaller its radius and the shorter the RP are. This is why the vortex pulse propagating in a medium with the anomalous GVD at larger distances more often refocuses. The behaviours of its temporal and spatial widths are depicted in Fig. $3 \mathrm{a}$.

It should be noted that inclusion of the GVD makes possible pulse refocusing for larger ratios $\alpha$ than those typical for the case when the dispersion is completely absent [8]. We would recall in this respect that the multifoci scenario observed in our previous work takes place at $\alpha=15.57$ or even smaller values. Moreover, refocusing and temporal reshaping processes can be controlled by both the magnitude and sign of such parameters as the collimation and the temporal chirp of the input pulse. So the magnitude and the sign of $(\mathrm{d} a / \mathrm{d} z)_{0}$ largely determine both the character and the period of the refocusing. As for the initial temporal chirp of the vortex pulse $(\mathrm{d} T / \mathrm{d} z)_{0}$, it can stop pulse shortening or stretching processes occurring in the medium at large enough values and turn them into the reverse processes. Such a scenario is illustrated in Fig. 3b, where one can see a non-monotonic dependence of the pulse duration $T(z)$ on the propagation distance in the medium with the anomalous dispersion for the temporal chirp of the input pulse $(\mathrm{d} T / \mathrm{d} z)_{0}=0.002$. Initially the pulse stretches and then it starts to shorten, beginning from the distance of $z=4 \mathrm{~mm}$. As a result, the RP increases up to $z=4 \mathrm{~mm}$ and then decreases.
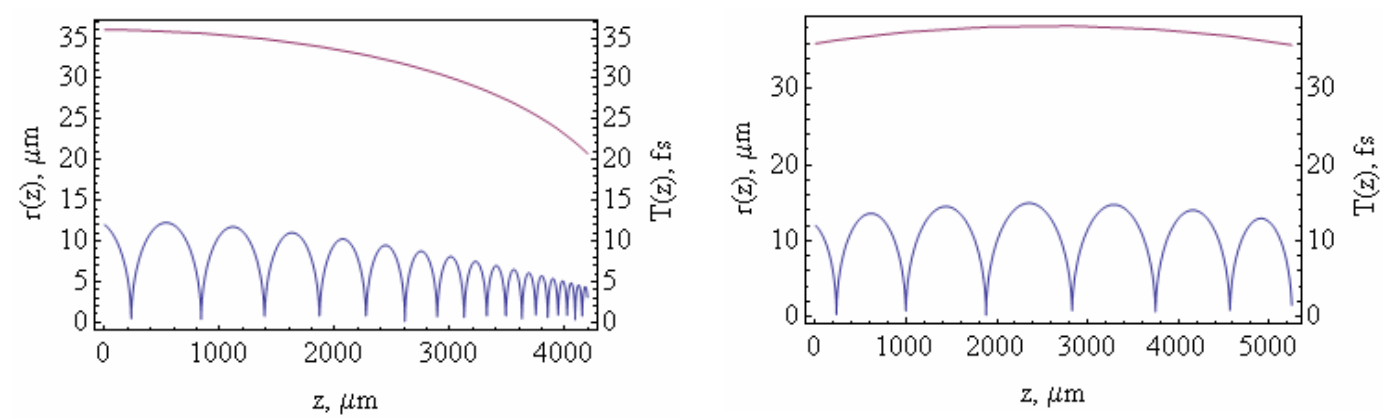

Fig. 3. Spatial dynamics of pulsed vortex beam radius $r(z)$ (lower curve) and duration $T(z)$ (upper curve) in a medium with anomalous GVD for $m=1, \alpha=29.52$ and the input parameters $a_{0}=12 \mu \mathrm{m}, T_{0}=36 \mathrm{fs}$, $\mathrm{da} / \mathrm{d} z(z=0)=-0.011, \lambda=1500 \mathrm{~nm}, I_{0}=97.1 \mathrm{TW} / \mathrm{cm}^{2}, \beta_{2}=-242 \mathrm{fs}^{2} / \mathrm{cm}:(a) \mathrm{dT} / \mathrm{d} z(z=0)=-0.00002$ and $(\mathrm{b})$ $\mathrm{d} T / \mathrm{d} z(z=0)=0.002$.

Ukr. J. Phys. Opt. 2013, Volume 14, Issue 2 
Being interested in the stable propagation of the pulsed vortex beams in the medium, we search for the soliton or, at least, quasi-soliton solutions. As earlier shown in the work [8], the competition of the Kerr focusing and the defocusing plasma nonlinearities can provide quasisoliton regime of the vortex propagation under negligible effect of the GVD. Fig. 1 corresponds exactly to this case. The GVD complicates searching for the soliton solutions. The solutions presented in Fig. 2 and Fig. 3 do not speak in favour of such scenarios, because the pulse durations do not remain constant under propagation. From our point of view, the combined effects of the diffraction, the Kerr nonlinearity, the GVD and the photoinduced plasma can lead to periodic oscillations not only of the pulse waist but also of its duration, providing stability of the pulse along the propagation axis. Currently we are in search of such scenarios. We are also interested in such regimes of the vortex pulse propagation when their parameters retain their initial values.

\section{Numerical simulations}

Now let us dwell upon the results of our numerical modelling. To gain detailed insight in various scenarios of the behaviour of powerful femtosecond pulses in Kerr dielectrics, we have solved numerically Eqs. (1) and (2) reduced to their dimensionless form (see Section 2), subject to the input parameters indicated above.

The modified NSE equation has been solved in the Cartesian coordinate system moving along with the group velocity. For numerical calculations, a splitting scheme has been used assuming that, at each small step along the pulse axis, the diffraction and dispersion effects are independent. The second-order differential operators in the spatial coordinates and the corresponding difference ones have been presented as locally one-dimensional operators. The resulting system of the difference equations has been solved with a sweep method along the appropriate spatial directions. The nonlinear terms have been involved, with the weight coefficients obeying the best summary approximation. This scheme is absolutely stable. To account for the dispersion, fast Fourier transform has been performed. Nonlinear dynamics of the pulsed vortex beams has been studied as a function of the input field parameters $\alpha, a_{0}, T_{0}$ and $m$. We have varied the ratio $\alpha$ from 12 to 20 .

We would like to emphasise that the GVD modifies significantly the propagation scenario for the vortex beams. Fig. 4a and Fig. 4b illustrate spatial evolution of $60 \mathrm{fs}$ pulse in the fused silica, when the topological charge is unit (the wavelength being $\lambda=800 \mathrm{~nm}$, the intensity $I_{0}=2.25 \mathrm{TW} \mathrm{cm}^{-2}$, and the initial width $\left.w_{0}=22 \mu \mathrm{m}\right)$. Here the ratio $\alpha$ is taken to be $\alpha=P_{\text {in }} / P_{\text {crit. }}=20$.

For the distance $z=1.5 L_{d f}$ (with $L_{d f}$ denoting the diffraction length) the pulse still retains its radial distribution and temporal profile (see Fig. 4a). However, transverse distortions and temporal reshaping occur for larger distances (beginning from $z=3.31 L_{d f}$ ). As our numerical analysis shows, then the three modulations affecting the vortex give rise to three filaments of different intensities. The final filaments rotate and do not fuse, since all of them must preserve the total angular momentum of the incident beam [15].

More complicated dynamics is inherent to the vortices with larger topological charges. For example, the vortex with the topological charge $m=2$ depicted in Fig. 5 undergoes reshaping of its temporal profile at smaller distances, when compared with those typical of singly charged vortices (already at $z=1.6 L_{d f}-$ see Fig. 5a). Simultaneously, transverse distortions begin to appear, leading to splitting of the doughnut beam into three subrings along the radial direction. Two peripheral subrings of lower intensities and a central subring of higher intensity then arise. The most 
severe distortions occur just in the central, most intense part of the doughnut beam. The filamentation process begins at the distance $z=2.4 L_{d f}$ (see Fig. 5b), which leads ultimately to five filaments of different intensities. Two filaments are observed in Fig. $5 \mathrm{c}$ in the plane $y=0$ at the distance $z=2.4 L_{d f}$.
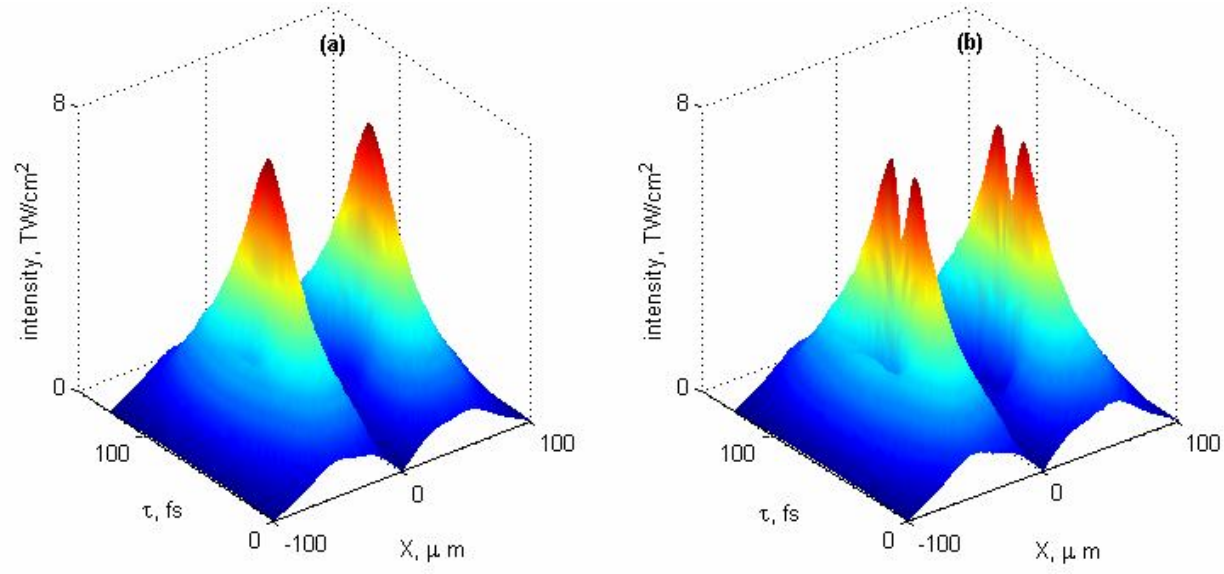

Fig. 4. Intensity profiles $I(x, t)$ of singly charged femtosecond vortex pulse in the plane $y=0$ at different propagation distances: (a) $z=1.5 L_{d f}$ and (b) $z=3.1 L_{d f}$.
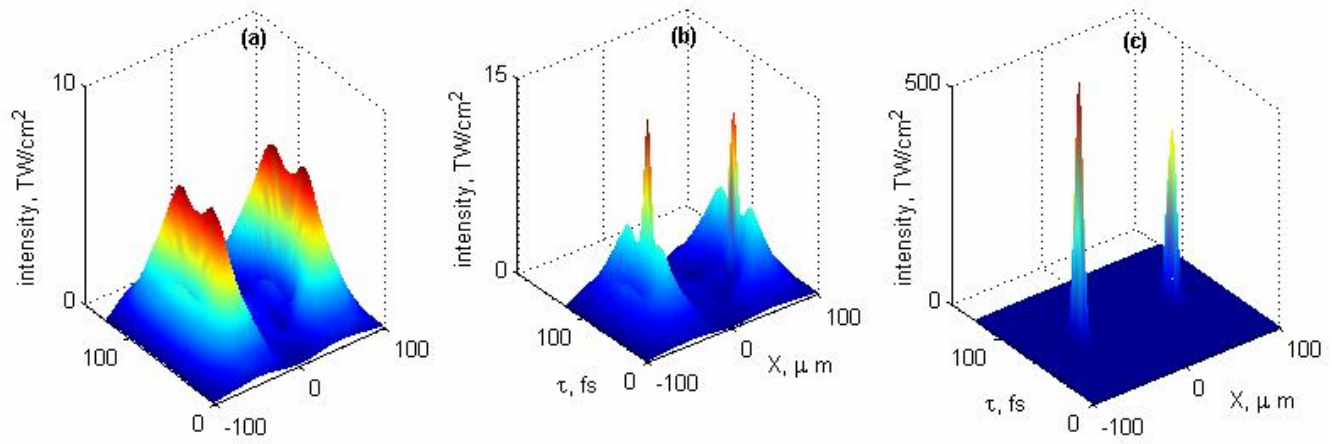

Fig. 5. Temporal reshaping and filamentation of vortex with $m=2$ at $\alpha=18$ and the same input parameters as in Fig.4: (a) spatio-temporal profile of vortex intensity in the plane $y=0$ at $z=1.6 L_{d f}$, (b) initial stage of filamentation at $z=2.4 L_{d f}$, and (c) two filaments in the plane $y=0$ at $z=2.8 L_{d f}$.

Let us proceed to a more detailed analysis of the vortex beam propagation at $m=3$ and $\alpha=12$ under combined effect of the factors mentioned above, supposing that the input pulse parameters are the same as earlier. Fig. 6 displays the earlier stages of vortex distortions. For the distance $z=0.8 L_{d f}$, the temporal envelope of the pulse distorts and a plateau is formed. At the same time, the radial structure of the vortex remains unaltered (see Fig. 6a) almost up to $z=1.2 L_{d f}$. At this distance the vortex undergoes reshaping of the temporal profile and the ring structure splits into three subrings. The outer and inner subrings of almost equal intensities still retain the original shape of the vortex. Reshaping is observed in the central part of the doughnut ring. While propagating, the distortions of the vortex accumulate, the inner ring grows in intensity (see Fig. 6c, Fig. $6 \mathrm{~d})$ and seven filaments arise at $z=2.2 L_{d f}$. 

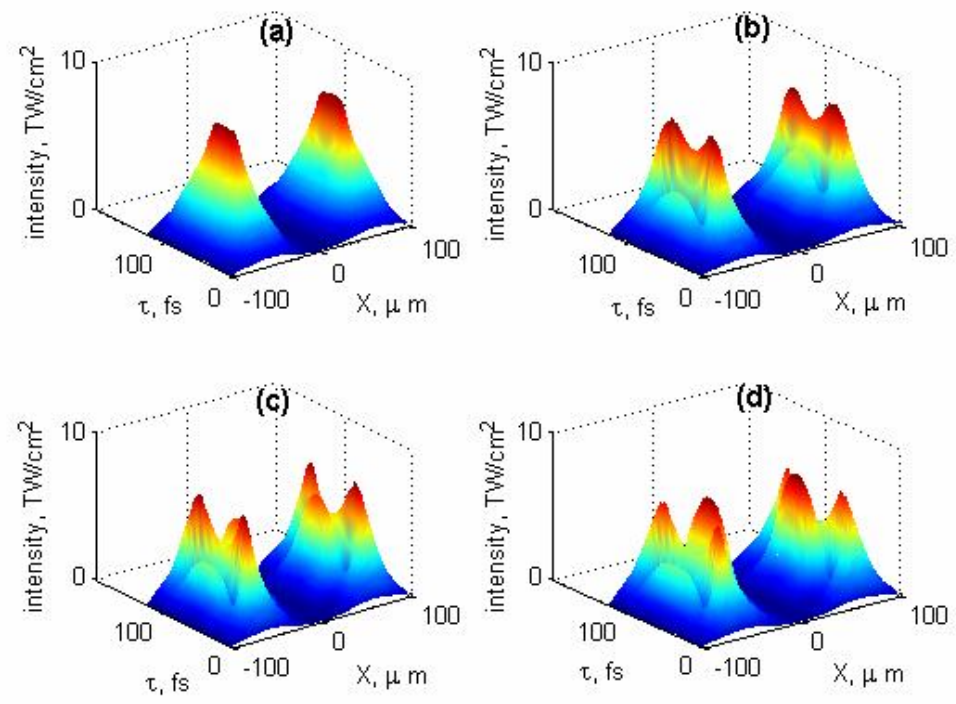

Fig. 6. Early stages of high-intensity femtosecond singular pulse filamentation for $m=3, \alpha=12$ and $y=0$ : (a) formation of plateau in the temporal profile at $z=0.8 L_{d f}$, (b) reshaping temporal envelope and ring splitting at $z=1.2 L_{d f}$, (c) accumulation of distortion at $z=1.6 L_{d f}$, and (d) intensity increase of the inner subring at $z=1.9 L_{d f}$

Notice that the problem considered here resembles to optical vortex bullet solutions. Generation of stable, fully three-dimensional, self-supported and long-lived light bullets having a large potential for various applications is one of grand challenges of the field of nonlinear optics, though it still remains open after several decades passed. The laser pulse cannot be trapped into spatiotemporal soliton in the normal-GVD media with the Kerr nonlinearity, because the evolution of this pulse results from the competition between the two main tendencies, the pulse compression in the transverse spatial direction as a result of self-focusing, and the pulse stretching along the time axis $T$. To overcome this obstacle, various approaches have been suggested, e.g. nonlinearity saturation [16], higher-order diffraction or dispersion [17], nonlocality of nonlinear response [18], and periodic (discrete) waveguide structures with controlled diffraction and/or GVD [19]. We suppose that the inertial character of defocusing nonlinearity of the photoinduced plasma in the Kerr dielectrics with the normal GVD can also provide a condition necessary for getting optical vortex bullets. The relevant results will be published in a forthcoming article.

\section{Conclusions}

In conclusion, in this work we have investigated the propagation of high-intensity femtosecond singular pulses in the Kerr medium, depending on the topological charge and the ratio of the initial pulse power to the critical one. The underlying physical processes governing the vortex propagation are the diffraction, the Kerr self-focusing and defocusing caused by the plasma generated via multiphoton ionisation process, and the GVD. For modelling we have employed a system involving the modified $(3+1)$-dimensional nonlinear Schrödinger equation and the kinetic equation for the free-electron density. The calculations have been based on semi-analytical and numerical approaches. The main results obtained can be formulated as follows.

When the pulsed vortex beam with above-critical input powers propagates through the Kerr medium under conditions of weak dissipation and dispersion, we deal with the competition of the 
focusing and defocusing factors, resulting in a periodic behaviour of the beam structure along the propagation axis. At the same time, the greater the topological charge, the less the period of beam refocusing becomes. Since the beam retains its shape, it would be right to say that it propagates in a quasi-soliton regime. When the impact of the GVD increases, the RP depends on the pulse duration and varies along the propagation axis in compliance with the changes in the pulse duration. The pulse elongates while propagating in the medium with the normal GVD, since the plasma defocusing does not compensate the dispersion effect. It leads to increasing RP. In the case of anomalous dispersion one can observe the reverse process. If the Kerr self-focusing increases the impact of the anomalous dispersion, the temporal width of the pulse decreases and, as a result, the pulse refocuses more often in the medium.

The early stages of filamentation of the pulsed vortex beam are described, which include reshaping of temporal profile of the vortex and splitting of the ring structure into three subrings along the radial direction. The outer and inner subrings of almost equal intensities still retain the original shape of the vortex. Reshaping is observed in the central part of the doughnut ring. When the vortex propagates, the filaments appear in the central ring, and the less intense peripheral rings disappear.

\section{Acknowledgement}

This work was supported by the Belarusian Republican Foundation for Fundamental Research (Grants F11-K-136 and F41.1/010).

\section{References}

1. Nye J F. and Berry M V, 1974. Dislocations in wave trains. Proc. R. Soc. London. Ser. A. 336: 165-190.

2. Soskin M and Vasnetsov M, 2001. Singular optics. Progress in Optics. 47: 221-276.

3. Twisted photons: Applications of light with orbital angular momentum, Ed. by J P Torres and L Torner, Willey -VCH (2011).

4. Shvedov V G, Desyatnikov A S, Rode A V, Izdebskaya Y V, Krolikowski W Z and Kivshar Y S, 2010. Optical vortex for trapping and transport of particles in air. Appl. Phys. A. 100: 327-331.

5. Desyatnikov A S, Torner L and Kivshar Y S, 2005. Optical vortices and vortex solitons. Progress in Optics. 47: 291-391.

6. Alexeyev C N, Volyar A and Yavorsky M A, Fiber optical vortices, in Lasers, Optics and Electro-Optics Research Trends, Ed. by L I Chen (Nova Science Pub., 2007), pp. 131-223.

7. Egorov Yu A, Fadeyeva T A and Volyar A V, 2004. Fine structure of singular beams in crystals: colours and polarization. J. Opt. A. 6: S217-S228.

8. Khasanov O, Smirnova T, Fedotova O, Rusetsky G and Romanov O, 2012. High-intensive femtosecond singular pulses in Kerr dielectrics. Appl. Opt. 51: 198-207.

9. Sudrie L, Couairon A, Franco M, Lamouroux B, Prade B, Tzortzakis S and Mysyrowitcz A, 2002. Femtosecond laser-induced damage and filamentary propagation in fused silica. Phys. Rev. Lett. 89: 186601-1-4.

10. Churilova A.V and Sukhorukov A P, 1999. Spatiotemporal effects at wave self-action in a dispersive medium with Kerr's nonlinearity. Bull. RAS, Physics. 63: 1913-1916.

11. Bergé L and Couairon A, 2000. Nonlinear propagation of self-guided ultra-short pulses in ionized gases. Phys. Plasmas. 7: 210-230.

Ukr. J. Phys. Opt. 2013, Volume 14, Issue 2 
12. Aközbek N, Bowden C M, Talebpour A and Chin S L, 2000. Femtosecond pulse propagation in air: variational analysis. Phys. Rev. E. 61: 4540-4549.

13. Wu Z, Jiang H, Yang H and Gong Q, 2003.The refocusing behaviour of a focused femtosecond laser pulse in fused silica. J. Opt. A: Pure Appl. Opt. 5: 102-107.

14. Henz S and Herrmann J, 1999. Self-focusing and pulse shortening of femtosecond pulses in multiphoton-ionized dispersive dielectric solids. Phys. Rev. A. 59: 2528-2531.

15. Vinçotte A and Bergé L, 2005. Femtosecond optical vortices in air. Phys. Rev. Lett. 95: 193901-1-14.

16. Blagoeva A B, Dinev S G, Dreischuh A A and Naidenov A, 1991. Light bullets formation in a bulk media. IEEE J. Quantum Electron. 27: 2060-2065.

17. Akhmediev N, Ankiewicz A and Soto-Crespo J M, 1993. Does the nonlinear Schrodinger equation correctly describe beam propagation? Opt. Lett. 18: 411-413.

18. Bang O, Krolikowski W, Wyller J and Rasmussen J J, 2002. Collapse arrest and soliton stabilization in nonlocal nonlinear media. Phys. Rev. E. 66: 046619.

19. Sukhorukov A A and Kivshar Y S, 2006. Slow-light optical bullets in arrays of nonlinear Bragg-grating waveguides. Phys. Rev. Lett. 97: 233901-4.

Khasanov O., Smirnova T., Fedotova O., Rusetsky G. and Volyar A., 2013. Powerful femtosecond singular pulses in the Kerr media with dispersion. Ukr.J.Phys.Opt. 14: 74 - 84.

Анотація. 3 використанням числового і варіаційного підходів проаналізована нелінійна динаміка високо інтенсивних фемтосекундних сингулярних імпульсів у керрівському середовищі з дисперсією групової швидкості. Вивчені фізичні прочеси, які керують поширенням вихорів: дифракиії, керрівського самофокусування, розфокусування завдяки фотоіндукованій плазмі і нормальної/аномальної дисперсії групових швидкостей. Виявлена багатофокусна поведінка вихорів у иьому середовищі. Показано, щзо період роз фокусування залежить, як від величини так $і$ від знаку дисперсї групових швидкостей. Описаний ранній етап філаментації вихрового променя. 